\title{
ИК-СПЕКТРОСКОПИЯ ПЛЕНОЧНОЙ СИСТЕМЫ Fе-ТіО, ПОЛУЧЕННОЙ МЕТОДОМ МАГНЕТРОННОГО РАСПЫЛЕНИЯ
}

\author{
() 2017 В. А. Логачева ${ }^{1}$, Н. Н. Афонин ${ }^{2}$, А. Н. Лукин ${ }^{1}$, Л. Н. Никитин ${ }^{3}$, Ю. А. Киселева ${ }^{1}$ \\ ${ }^{1}$ Воронежский государственный университет, Университетская пл., 1, 394018 Воронеж, Россия \\ e-mail:kcmf@vsu.ru \\ ${ }^{2}$ Воронежский государственный педагогический университет, ул. Ленина, 86, 394043 Воронеж, Россия \\ e-mail:nafonin@vspu.ac.ru \\ ${ }^{3}$ Воронежский государственный технический университет, Московский пр., 14, 394026 Воронеж, Россия
}

Поступила в редакцию 17.04.2017 г.

\begin{abstract}
Аннотация. Проведен сравнительный анализ оптических спектров отражения-поглощения (RAS) и нарушенного полного внутреннего отражения (ATR) пленочной системы $\mathrm{Fe}-\mathrm{TiO}_{2}$, полученной методом реактивного магнетронного распыления с последующим отжигом в вакууме и при атмосферном давлении в потоке кислорода. Показано, что смещение характеристических колебаний связи Тi-О в сторону меньших частот связано с присутствием в кристаллической структуре $\mathrm{TiO}_{2}$ катионов $\mathrm{Fe}^{+3}$. Установлено, что полосы поглощения, обусловленные деформационными колебаниями связи $\mathrm{Fe}-\mathrm{OH}$, наблюдаются только в спектрах ATR и смещены в сторону больших частот по сравнению с индивидуальными оксидами железа, что свидетельствует об образовании сложных оксидов в пленочной системе $\mathrm{Fe}-\mathrm{TiO}_{2}$.
\end{abstract}

Ключевые слова: ИК-спектроскопия, магнетронное распыление, пленочная система $\mathrm{Fe}-\mathrm{TiO}_{2}$.

\section{ВВЕДЕНИЕ}

Диоксид титана находит широкое применение в составе диэлектрических, защитных, самоочищающихся покрытий [1], как фотокатализатор для бытовой и промышленной очистки воды и воздуха [2], компонент сенсорных датчиков [3]. Однако его ширина запрещенной зоны составляет 3-3.2 эВ (в зависимости от кристаллической фазы), поэтому он проявляет значительную фотокаталитическую активность только при облучении ультрафиолетовым излучением с длиной волны меньше 400 нм, a, следовательно, проведение фотокаталитических реакций с применением $\mathrm{TiO}_{2}$ требует использования специальных источников ультрафиолетового излучения. Расширение спектра поглощения в видимую область позволило бы использовать солнечное излучение. Одним из подходов, используемых для изменения оптических свойств $\mathrm{TiO}_{2}$, является легирование оксида титана переходными металлами.

В ряде работ диоксид титана легировали ионами различных щелочноземельных металлов: $\left(\mathrm{Ca}^{2+}\right.$, $\mathrm{Sr}^{2+}$ и $\mathrm{Ba}^{2+}$ ) [4]; переходных металлов: $\mathrm{Fe}^{3+}, \mathrm{Cr}^{6+}$ [5], $\mathrm{Sn}^{4+}[6]$, а также ионами редкоземельных элементов: $\left(\mathrm{La}^{3+}, \mathrm{Ce}^{3+}, \mathrm{Er}^{3+}, \mathrm{Pr}^{3+}, \mathrm{Nd}^{3+}, \mathrm{Sm}^{3+}\right)$ [7]. Ионы ме- таллов создают энергетические состояния в запрещенной зоне $\mathrm{TiO}_{2}$, которые могут вызывать светочувствительные переходы при облучении в видимом диапазоне длин волн из-за возбуждения электрона из этого уровня энергии в зону проводимости $\mathrm{TiO}_{2}$.

Сдвиг фундаментального края поглощения в легированных металлами оксидных системах имеет сложную природу. Он может быть вызван однородным замещением катионов $\mathrm{Ti}^{4+}$ легирующей примесью, и в этом случае будет зависеть от концентрации, которая определяется пределом растворимости легирующего металла в $\mathrm{TiO}_{2}$. Если сдвиг края поглощения зависит от температуры отжига, он может быть связан с образованием и сегрегированием кластеров $\mathrm{M}_{x} \mathrm{O}_{y}$ [8]. В то время как природа сдвига не важна в оптических применений этих систем (например, в качестве оптических фильтров), она имеет решающее значение для объяснения природы фотокаталитической активности структур $\mathrm{M}^{\mathrm{n}+}-\mathrm{TiO}_{2}$

Особый интерес представляют системы $\mathrm{Fe}-$ $\mathrm{TiO}_{2}$ по нескольким причинам: 1- катионы железа оказывают большое влияние на время рекомбинации носителей заряда; 2 - присутствие железа ин- 
дуцирует батохромный эффект; 3 - легирование железом позволяет использовать оксид титана в важных фотокаталитических реакциях восстановления и окисления.

Легирование железом частиц $\mathrm{TiO}_{2}$ приводит к образованию смешанных оксидов или смеси простых и смешанных оксидов. Катионы железа $\mathrm{Fe}^{3+}$ замещают $\mathrm{Ti}^{+4}$ в катионных позициях, так как имеют одинаковые ионные радиусы $\mathrm{Fe}^{+3}(0.64 \AA), \mathrm{Ti}^{+4}$ $(0.61 \AA$ ) [9] и образуют твердые растворы с диоксидом титана при низких концентрациях железа ( $<1 \%$ ) [10]. Присутствие железа снижает температуру превращения анатаза в рутил до $400{ }^{\circ} \mathrm{C}$ [11]. Образование твердых растворов и существование пределов растворимости для ионов $\mathrm{Fe}$ в $\mathrm{TiO}_{2}$ приводят к образованию $\alpha-\mathrm{Fe}_{2} \mathrm{O}_{3}$ при более высоких концентрациях железа. В зависимости от применяемой термической обработки и содержания железа могут образовываться в системе $\mathrm{Fe}-\mathrm{Ti}$ различные смешанные оксиды: $\mathrm{Fe}_{2} \mathrm{TiO}_{5}$ (псевдобрукит), $\mathrm{Fe}_{2} \mathrm{Ti}_{3} \mathrm{O}_{9}$ (псевдорутил) и $\mathrm{Fe}_{2} \mathrm{Ti}_{2} \mathrm{O}_{7}$, включая $\alpha-\mathrm{Fe}_{2} \mathrm{O}_{3}$, $\mathrm{FeTiO}_{3}$ и $\mathrm{Fe}_{x}(\mathrm{Ti})_{1-0.75 x} \mathrm{O}_{2-\delta}(0.01<x<0.14)$. Замещение титана на $\mathrm{Fe}^{3+}$ в $\mathrm{TiO}_{2}$ существенно влияет на рекомбинацию носителей заряда. Некоторые исследования показывают, что Fe (III) ведет себя как электрон/дырочный центр рекомбинации. Другие указывают, что легирование до 0.5 ат.\% $\mathrm{Fe}^{3+}$ резко увеличивает время жизни носителей заряда, которое может достигать нескольких минут и даже часов (в собственном $\mathrm{TiO}_{2}$ среднее время жизни пары электрон-дырка составляет около 30 нс) [10]. Кроме того, ионы железа снижают скорость кристаллизации $\mathrm{TiO}_{2}$, что приводит к уменьшению размера частиц [12], а также усиливают гидрофильный характер катализатора.

В связи с этим представляет большой практический интерес разработка методов модификации оптических свойств оксида титана. Целью работы было выявление образования сложных оксидов методом ИК-спектроскопии на поверхности и внутри пленочной системы $\mathrm{Fe}-\mathrm{TiO}_{2}$, полученной реактивным магнетронным распылением с последующим отжигом в вакууме при разном давлении и термооксидировании при атмосферном давлении в потоке кислорода.

\section{МЕТОДИКА ПРОВЕДЕНИЯ ЭКСПЕРИМЕНТА}

На пластинах монокристаллического кремния с подслоем платины (110 нм) методом магнетронного распыления осаждали пленку металлического титана толщиной 100 нм. Для получения оксида титана осуществляли термообработку в печи резистивного нагрева в потоке кислорода при $T=973 \mathrm{~K}$ в течение 30 минут.

Модифицирование пленок оксида титана проводили путем магнетронного распыления железа в модернизированной установке УВН-1 в плазме аргона при режимах: давление в камере $13.3 \cdot 10^{-2}$ Па, ток разряда $0.5 \mathrm{~A}$, напряжение разряда $420 \mathrm{~B}$, с последующим отжигом сформированной пленочной системы $\mathrm{Fe}-\mathrm{TiO}_{2} / \mathrm{Pt} / \mathrm{Si}$ в вакууме и при атмосферном давлении в потоке кислорода. Материалом катода служила мишень металлического железа с содержанием примесей не более 0.01 ат.\%. Скорость распыления железа составила 7.2 нм/мин, толщина пленки задавалась временем распыления и составляла от 90 до 120 нм. Отжиг пленочных образцов осуществляли в кварцевых ампулах, откаченных до давления $P=10^{-4}$ Па, и в вакуумной камере дифрактометра ARL X'TRA при давлении $P=1.33 \cdot 10^{-2}$ Па, термооксидирование - в потоке кислорода в кварцевой печи резистивного нагрева при $T=1073$ К в течение 30 мин.

Для исследования образования сложных оксидов в пленочной системе $\mathrm{Fe}-\mathrm{TiO}_{2}$ был использован метод ИК-спектроскопии, который позволяет изучать строение многослойных тонкопленочных систем, при этом колебательные спектры решетки от различных слоев наблюдаются как совмещение спектров каждого слоя. Поскольку колебания решетки очень чувствительны к ближайшим атомам, использование ИК-спектроскопии дает дополнительную, новую и иногда более подробную информацию относительно свойств и качеств тонких пленок в сравнении с рентгеновской дифракцией.

Измерение спектров отражения-поглощения (RAS) и нарушенного полного внутреннего отражения (ATR) пленочной системы проводилось на инфракрасном Фурье спектрометре Vertex-70 фирмы Брукер (Германия). Для измерения спектров отражения - поглощения использовалась приставка зеркального отражения с переменным углом падения $13-83^{\circ}$. Для измерения спектров ATR использовалась одноходовая приставка Platinum-ATR c алмазной призмой.

Ha спектрах RAS от пленочных образцов $\mathrm{Fe}-$ $\mathrm{TiO}_{2} / \mathrm{Pt} / \mathrm{Si}$, снятых под углом $13^{\circ}$, на блюдается картина, характерная для спектров отражения - поглощения, т.е. помимо спектра отражения от поверхности, наблюдается спектр поглощения луча прошедшего через оксидный слой и отразившегося от металлического подслоя Pt, лежащего на монокристаллической подложке кремния. Таким образом, на 
данных спектрах наблюдаются полосы поглощения комплексами (группами молекул) образовавшимися по всей глубине слоя.

Спектры ATR были получены при механическом прижатии образцов к алмазной призме с усилием около 1 кГс. При данном методе полосы поглощения на спектре ATR связаны с поверхностными состояниями. Спектры ATR для наглядности, были преобразованы в спектры пропускания.

\section{РЕЗУЛЬТАТЫ И ИХ ОБСУЖДЕНИЕ}

На рис. 1 представлены ИК-спектры ATR для пленок: полученных магнетронным распылением железа на оксид титана, кривая 1; отжигом в вакуумной камере дифрактометра при $P=1.33 \cdot 10^{-2}$ Па, кривая 2; вакуумным восстановительном отжигом при $P=10^{-4}$ Па, кривая 3; термооксидированием в потоке кислорода, кривая 4. Все отжиги проводили при $T=1073$ К в течение 30 мин.

Кривая 1 без характерных полос поглощения принадлежит спектру ATR от металлической пленки железа. Пленки, полученные при разных режимах отжига, были гетерофазными по составу и со- держали $\mathrm{TiO}_{2}$ в структуре рутила, два оксида железа: $\mathrm{Fe}_{3} \mathrm{O}_{4}$ и $\mathrm{Fe}_{2} \mathrm{O}_{3}$, и фазы сложных оксидов на основе оксидов титана и железа: $\mathrm{FeTiO}_{3}$ (ильменит) и $\mathrm{Fe}_{2} \mathrm{TiO}_{4}$ (ульвошпинель) [13], характерные полосы поглощения которых наблюдаются на кривых 2-4. Для оксида титана в структуре рутила полосы валентных колебаний Ті-О в индивидуальных оксидах лежат в области края собственного поглощения 400-800 см-1 [14]. Колебания связи металл-кислород на поверхности в соответствии с [15] характеризуются полосами поглощения 770 и $730 \mathrm{~cm}^{-1}$. На кривых 3, 2 после вакуумного отжига с увеличением степени восстановления происходит разрыв связей титан-кислород и наблюдается увеличение интенсивности отражения (уменьшение поглощения) полосы при $767 \mathrm{~cm}^{-1}$. Сдвиг полосы отражения в высокочастотную область с $722 \mathrm{~cm}^{-1}$ (для окисленной пленки) до $767 \mathrm{~cm}^{-1}$ (для восстановленной) на $45 \mathrm{~cm}^{-1}$ свидетельствует о значительной разнице в энергетике связи Ті-О на поверхности по сравнению с объемом пленки. Кроме того, сдвиг полосы поглощения в высокочастотную область при восстановительном отжиге свидетельствует о взаи-

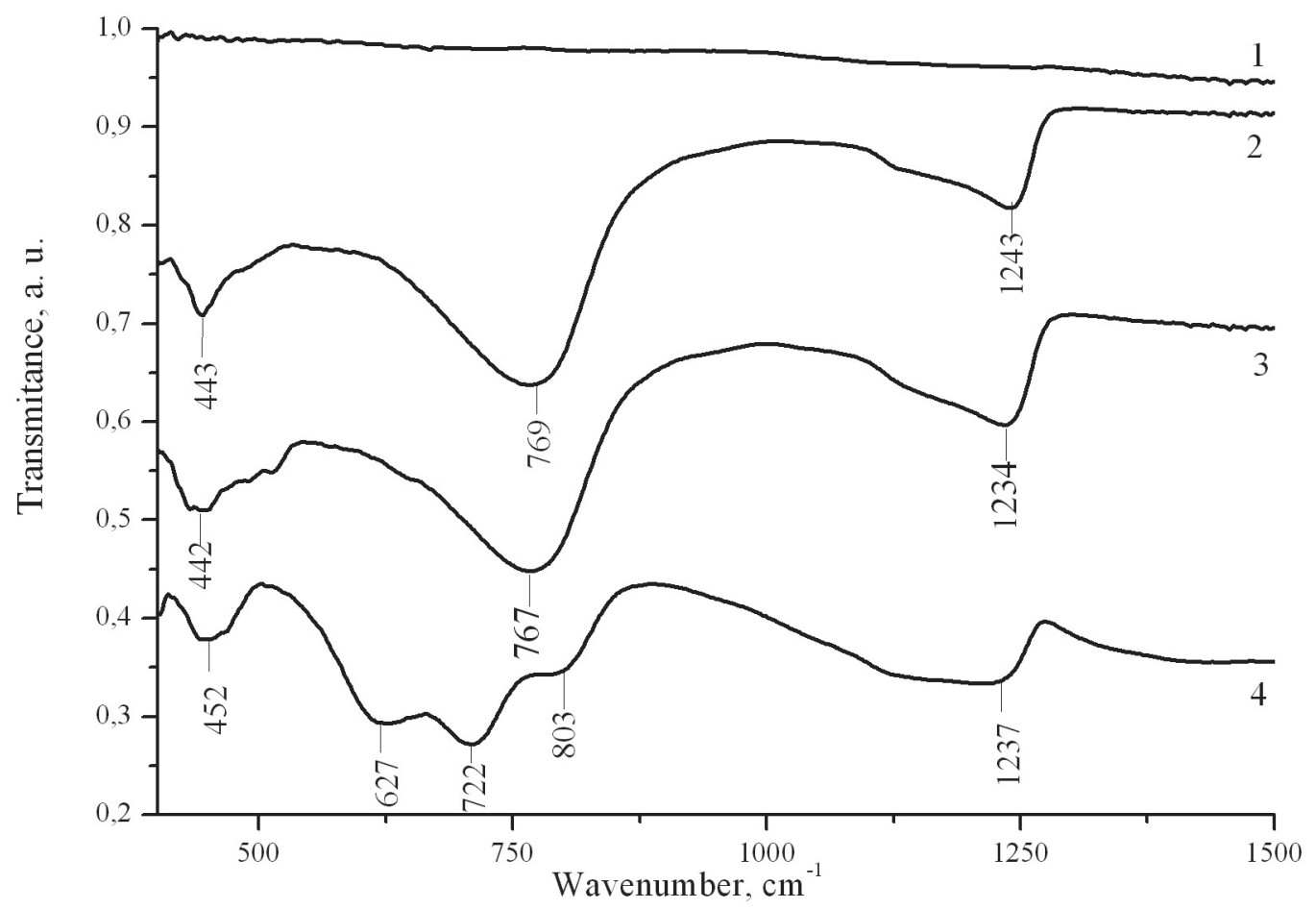

Рис. 1. ИК-спектры ATR пленочных образцов системы $\mathrm{Fe}-\mathrm{TiO}_{2} / \mathrm{Pt} / \mathrm{Si}$, полученных: 1 - магнетронным распылением железа на оксид титана; 2 - отжигом в вакуумной камере дифрактометра при $P=1.33 \cdot 10^{-2}$ Па; 3 - вакуумным восстановительном отжигом при $P=10^{-4}$ Па; 4 - термооксидированием в потоке кислорода

[Fig. 1. IR spectra of ATR of the $\mathrm{Fe}-\mathrm{TiO}_{2} / \mathrm{Pt} / \mathrm{Si}$ film system obtained by: 1 - magnetron sputtering of iron on titanium oxide; 2 - annealing in a vacuum chamber of a diffractometer at $P=1.33 \cdot 10^{-2} \mathrm{~Pa} ; 3$ - vacuum reduction annealing at $P=10^{-4} \mathrm{~Pa} ; 4$ - thermooxidation in flowing oxygen] 
модействии атомов кислорода с координационноненасыщенными атомами титана на поверхности. Интенсивность этой полосы поглощения вновь восстанавливается при высокотемпературном взаимодействии с кислородом, кривая 4.

Полосы, характеризующие валентные колебания связи Тi-O 803 и 452 cм$^{-1}\left(831\right.$ и 458 cm$^{-1}$ согласно [14]), и полосы колебания связи $\mathrm{Fe}-\mathrm{O} 627 \mathrm{~cm}^{-1}$ в $\alpha-\mathrm{Fe}_{2} \mathrm{O}_{3}\left(662 \mathrm{~cm}^{-1}[14]\right)$, наблюдаются только на спектре после окислительного отжига (кривая 4).

Полоса поглощения $1237 \mathrm{~cm}^{-1}$ характеризует колебания связей металл-гидроксидная группа на поверхности пленки и принадлежит деформационным колебания связи $\mathrm{Fe}-\mathrm{OH}$ [16]. Её интенсивность существенно снижается при вакуумном отжиге.

Поверхность пленки отличается в значительной степени от структуры объема пленки. Атомы на поверхности имеют меньшее число ближайших соседей, поэтому энергия их связи отличается от энергии связи атомов в объеме. Этим фактом обусловлено появление на поверхности колебаний, которые дают дополнительные линии в ИК-спектрах поглощения. Введение атомов примеси приводит к возникновению локальных колебаний. ИК-спектроскопия может дать прямую информацию о колебаниях связей поверхностный катион-кислород.
Сравнение ИК-спектров RAS, кривые 1 и ATR, кривые 2, приведено на рис. 2-4. В табл. 1-3 выполнено отнесение полос поглощения на основе литературных данных.

ИК-спектры RAS и ATR свидетельствуют об образовании оксидных фаз железа и титана в пленке, полученной после отжига при $P=10^{-4}$ Па. Наблюдаемые полосы поглощения $650 \mathrm{~cm}^{-1}$ (спектр отражения-поглощения, кривая 1) и $626 \mathrm{~cm}^{-1}$ (спектр пропускания, кривая 2) могут быть отнесены к валентным колебаниям связей $\mathrm{Fe}-\mathrm{O}$ в $\alpha-\mathrm{Fe}_{2} \mathrm{O}_{3}$, рис. 2 . Полосы поглощения $458 \mathrm{~cm}^{-1}$ (кривая 1) и $451 \mathrm{~cm}^{-1}$ (кривая 2) характеризуют колебания связи Тi-O в оксиде титана в структуре рутила. Только для этой пленки и на поверхности, и в объеме наблюдаются полосы поглощения в области 1261 и $1224 \mathrm{~cm}^{-1}$. Они могут быть отнесены к колебаниям связи $\mathrm{Fe}-\mathrm{OH}$, табл. 1. Наблюдаемое смещение частоты колебаний в высокочастотную область свидетельствует об уменьшение координационного числа $\mathrm{Ti}^{+4}$ в объеме пленки при образовании сложных оксидов на основе оксида железа и рутила.

На рис. 3. представлены ИК-спектры, полученные для пленки, отожженной при $P=1.33 \cdot 10^{-2}$ Па, в табл. 2. показано соотнесение полос поглощения. На ИК-спектре ATR (кривая 2) наблюдается значи-

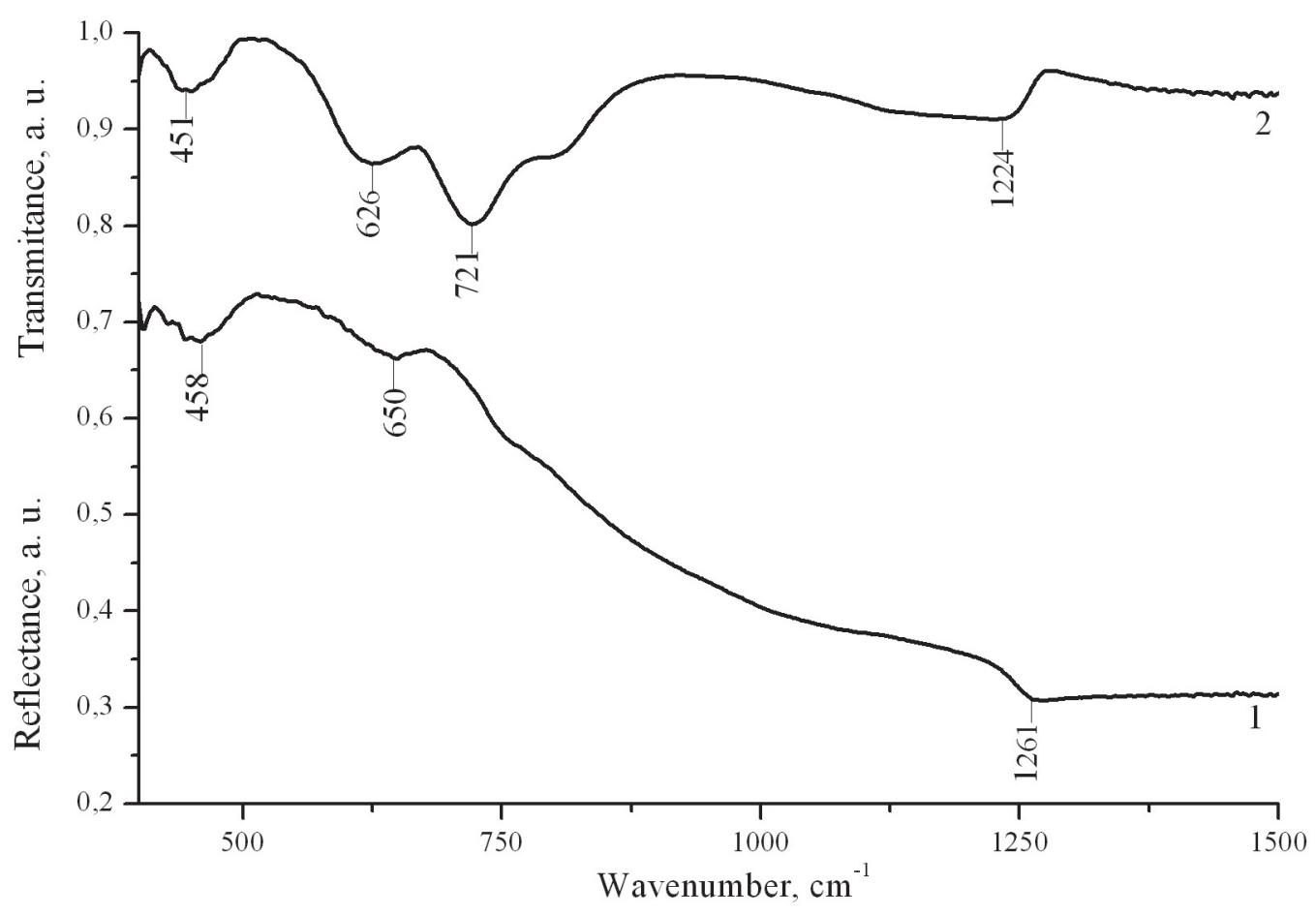

Рис. 2. ИК-спектры пленочного образца системы $\mathrm{Fe}-\mathrm{TiO}_{2} / \mathrm{Pt} / \mathrm{Si}$, полученного отжигом при $P=10^{-4}$ Па: 1 - RAS; 2 - ATR

[Fig. 2. IR spectra of the $\mathrm{Fe}-\mathrm{TiO}_{2} / \mathrm{Pt} / \mathrm{Si}$ film system obtained by annealing at $P=10^{-4} \mathrm{~Pa}: 1-\mathrm{RAS} ; 2-\mathrm{ATR}$ ] 


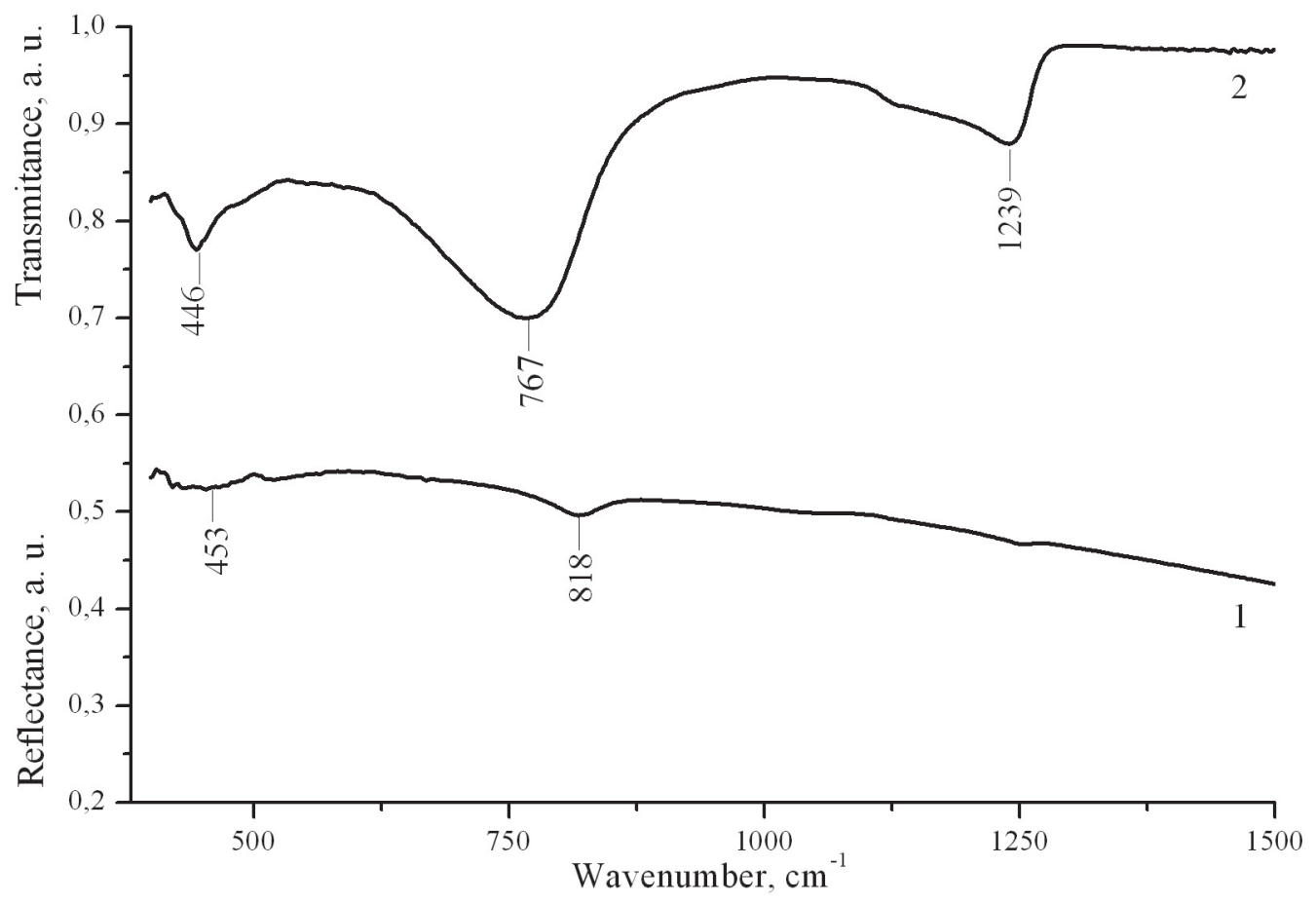

Pис. 3. ИК-спектры пленочного образца системы $\mathrm{Fe}-\mathrm{TiO}_{2} / \mathrm{Pt} / \mathrm{Si}$, полученного вакуумным восстановительном отжигом при $P=1.33 \cdot 10^{-2}$ Па: 1 - RAS; 2 - ATR

[Fig. 3. IR spectra of the $\mathrm{Fe}-\mathrm{TiO}_{2} / \mathrm{Pt} / \mathrm{Si}$ film system obtained by vacuum reduction annealing at $\left.P=1.33 \cdot 10^{-2} \mathrm{~Pa}: 1-\mathrm{RAS} ; 2-\mathrm{ATR}\right]$

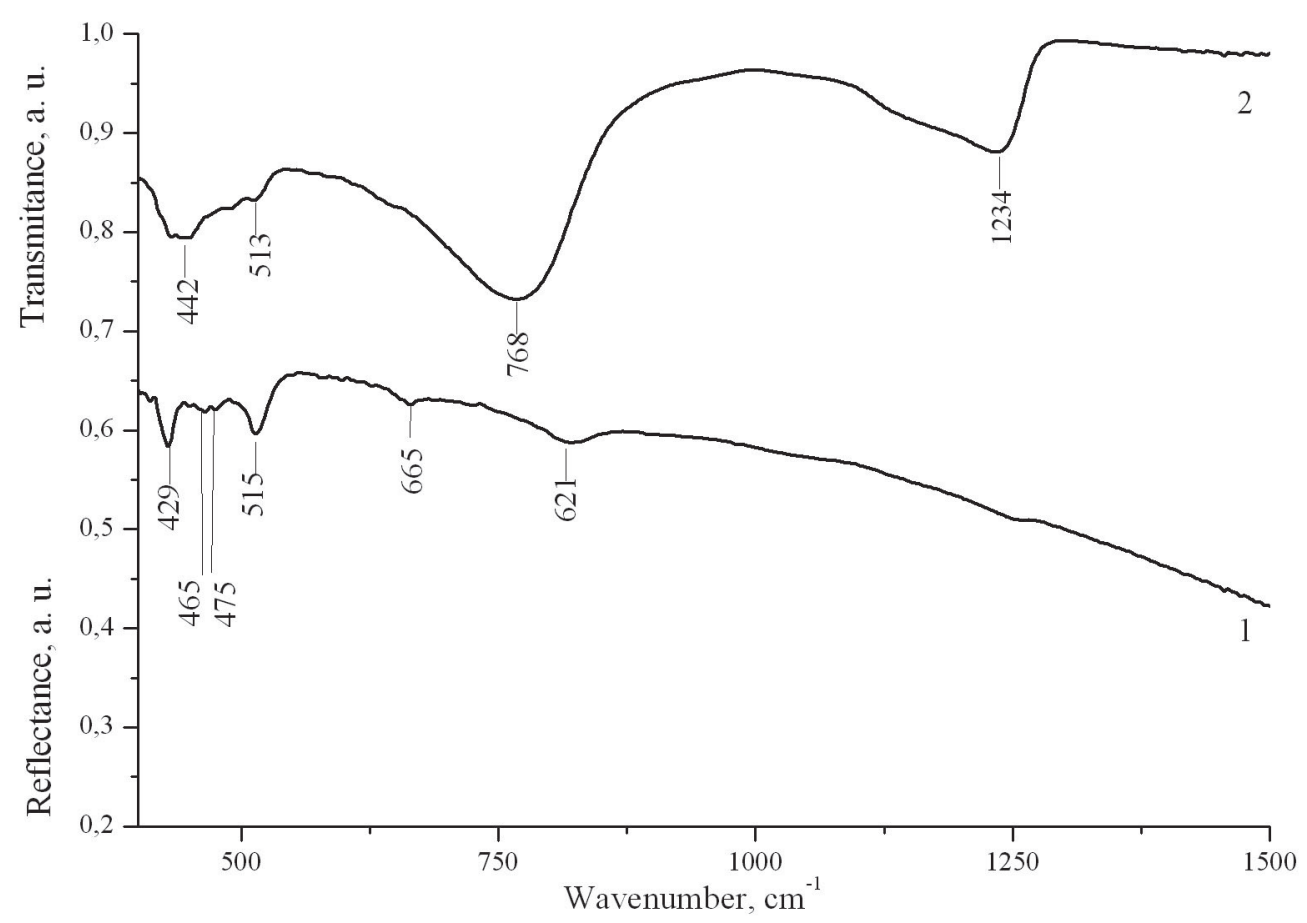

Рис. 4. ИК-спектры пленочного образца системы $\mathrm{Fe}-\mathrm{TiO}_{2} / \mathrm{Pt} / \mathrm{Si}$, полученного термооксидированием в потоке кислорода: 1 - RAS; 2 - ATR

[Fig. 4. IR spectra of the $\mathrm{Fe}-\mathrm{TiO}_{2} / \mathrm{Pt} / \mathrm{Si}$ film system obtained by thermoxidation in flowing oxygen: 1 - RAS; 2 - ATR] 
тельный рост интенсивности поглощения линий, характеризующий валентные колебания связи Ті-О: 767 и $446 \mathrm{~cm}^{-1}$, а также присутствует полоса поглощения, характеризующая деформационные колебания связи $\mathrm{Fe}-\mathrm{OH}, 1239 \mathrm{~cm}^{-1}$. В ИК-спектре RAS (кривая 1) отсутствует полоса поглощения, которую можно отнести к колебаниям связи $\mathrm{Fe}-\mathrm{OH}$.

После термооксидирования в потоке кислорода в ИК-спектре RAS (кривая 1, рис. 4) пленки наблюдаются новые полосы поглощения, характеризующие валентные колебания связи $\mathrm{Fe}-\mathrm{O}$ в $\alpha-\mathrm{Fe}_{2} \mathrm{O}_{3}$ : $665,515,429 \mathrm{~cm}^{-1}$, (табл. 3). Колебания связи $\mathrm{Fe}-\mathrm{OH}$ с частотой $1234 \mathrm{~cm}^{-1}$ наблюдаются только в спектрах ATR, характеризующих поверхность пленки (кривая 2, рис. 4).

\section{ЗАКЛЮЧЕНИЕ}

Проведенный сравнительный анализ спектров нарушенного полного внутреннего отражения (ATR) со спектрами отражения - поглощения (RAS) показал, что характер ИК-спектров зависит от давления, при котором производился отжиг пленок чем выше давление, тем больше наблюдается полос поглощения, относящихся к индивидуальным оксидам: $\alpha-\mathrm{Fe}_{2} \mathrm{O}_{3}$ и $\mathrm{TiO}_{2}$. Частота колебаний этих полос смещается в коротковолновую область для спектров ATR. Полосы поглощения, обусловленные деформационными колебаниями $\mathrm{Fe}-\mathrm{OH}$, наблюдаются только в спектрах ATR и смещены в сторону больших частот по отношению к соответствующим значениям полос поглощения для индивидуальных оксидов железа $\left(1045 \mathrm{~cm}^{-1}\right)$. Это может свидетельствовать об образовании сложных оксидов в пленочной системе $\mathrm{Fe}-\mathrm{TiO}_{2}$ - ильменита $\mathrm{FeTiO}_{3}$ и ульвошпинели $\mathrm{Fe}_{2} \mathrm{TiO}_{4}$, что согласуется с данными дифрактометрических исследований фазового состава этих пленок. Наблюдаемые полосы поглощения в спектрах пропускания 1233-1242 см-1 можно отнести к колебаниям связей М-О в этих сложных оксидах.

Таблица 1. Максимумы полос поглощения $\left(\mathrm{cm}^{-1}\right)$ и их отнесение в ИК-спектрах пленочного образца системы $\mathrm{Fe}-\mathrm{TiO}_{2} / \mathrm{Pt} / \mathrm{Si}$ после вакуумного отжига при $P=10^{-4}$ Па

[Table 1. Maximum absorption wavenumbers $\left(\mathrm{cm}^{-1}\right)$ and their attribution in the IR spectra of the $\mathrm{Fe}-\mathrm{TiO}_{2} / \mathrm{Pt} / \mathrm{Si}$ film system after vacuum annealing at $\left.P=10^{-4} \mathrm{~Pa}\right]$

\begin{tabular}{|c|c|c|c|}
\hline $\begin{array}{c}\text { Связи } \\
\text { [Bonds] }\end{array}$ & $\begin{array}{c}\text { Моды } \\
\text { Поверхность пленки } \\
\text { [Modes, } \\
\text { Surface of the film] } \\
\text { ATR }\end{array}$ & $\begin{array}{c}\text { Моды } \\
\text { Объем пленки } \\
\text { [Modes, } \\
\text { Volume of the film] } \\
\text { RAS }\end{array}$ & $\begin{array}{l}\text { Литература } \\
\text { [References] }\end{array}$ \\
\hline $\mathrm{Fe}-\mathrm{OH}$ & 1224 & 1261 & $1121[16]$ \\
\hline $\mathrm{Fe}_{2} \mathrm{O}_{3}$ & - & 650 & 655 [14] \\
\hline Ti-O & 721 & - & 730 [15] \\
\hline$\alpha-\mathrm{Fe}_{2} \mathrm{O}_{3}$ & 626 & - & 655 [14] \\
\hline vTi-O-Ti в $\mathrm{TiO}_{2}$ & 451 & 458 & 458 [14] \\
\hline
\end{tabular}

Таблица 2. Максимумы полос поглощения $\left(\mathrm{cm}^{-1}\right)$ и их отнесение в ИК-спектрах пленочного образца системы $\mathrm{Fe}-\mathrm{TiO} / \mathrm{Pt} / \mathrm{Si}$ после вакуумного отжига при $P=1.33 \cdot 10^{-2}$ Па

[Table 2. Maximum absorption wavenumbers $\left(\mathrm{cm}^{-1}\right)$ and their attribution in the IR spectra of the $\mathrm{Fe}-\mathrm{TiO} / \mathrm{Pt} / \mathrm{Si}$ film system after annealing at $\left.P=1.33 \cdot 10^{-2} \mathrm{~Pa}\right]$

\begin{tabular}{|c|c|c|c|}
\hline $\begin{array}{c}\text { Связи } \\
{[\text { Bonds }]}\end{array}$ & $\begin{array}{c}\text { Моды } \\
\text { Поверхность пленки } \\
\text { [Modes, } \\
\text { Surface of the film] } \\
\text { ATR }\end{array}$ & $\begin{array}{c}\text { Моды } \\
\text { Объем пленки } \\
\text { [Modes, } \\
\text { Volume of the film] } \\
\text { RAS }\end{array}$ & $\begin{array}{c}\text { Литература } \\
\text { [References] }\end{array}$ \\
\hline $\mathrm{Fe}-\mathrm{OH}$ & 1239 & - & $1121[16]$ \\
\hline$v \mathrm{Ti}-\mathrm{O}-\mathrm{Ti}$ & 767 & - & $760[17]$ \\
\hline $\mathrm{TiO}$ в TiO & 446 & - & $468,410[17]$ \\
\hline $\mathrm{Ti}-\mathrm{O}$ & - & 453 & $800[18]$ \\
\hline $\mathrm{Ti}-\mathrm{O}$ в $\mathrm{TiO}_{2}$ & - & & $468,410[17]$ \\
\hline
\end{tabular}


Таблица 3. Максимумы полос поглощения $\left(\mathrm{cm}^{-1}\right)$ и их отнесение в ИК-спектрах пленочного образца системы $\mathrm{Fe}-\mathrm{TiO}_{2} / \mathrm{Pt} / \mathrm{Si}$ после термооксидирования

[Table 3. Maximum absorption wavenumbers $\left(\mathrm{cm}^{-1}\right)$ and their attribution in the IR spectra of the $\mathrm{Fe}-\mathrm{TiO}_{2} / \mathrm{Pt} / \mathrm{Si}$ film system after thermoxidation]

\begin{tabular}{|c|c|c|c|}
\hline $\begin{array}{c}\text { Связи } \\
{[\text { Bonds] }}\end{array}$ & $\begin{array}{c}\text { Моды } \\
\text { Поверхность пленки } \\
\text { [Modes, } \\
\text { Surface of the film] } \\
\text { ATR }\end{array}$ & $\begin{array}{c}\text { Моды } \\
\text { Объем пленки } \\
\text { [Modes, } \\
\text { Volume of the film] } \\
\text { RAS }\end{array}$ & $\begin{array}{c}\text { Литература } \\
\text { [References] }\end{array}$ \\
\hline $\mathrm{Fe}-\mathrm{OH}$ & 1234 & - & $1121[16]$ \\
\hline$v \mathrm{Ti}-\mathrm{O}-\mathrm{Ti}$ & 768 & - & $760[17]$ \\
\hline$\alpha-\mathrm{Fe}_{2} \mathrm{O}_{3}$ & 513 & - & $524[14]$ \\
\hline$v \mathrm{Ti}-\mathrm{O}-\mathrm{Ti}$ & 442 & - & $468[17]$ \\
\hline $\mathrm{Ti}^{-\mathrm{O}}$ & - & 821 & $800[18]$ \\
\hline$\alpha \mathrm{Fe} \mathrm{O}_{3}$ & - & 665 & $655[14]$ \\
\hline$\alpha \mathrm{Fe} \mathrm{O}_{3}$ & - & 515 & $524[14]$ \\
\hline$v \mathrm{Ti}-\mathrm{O}-\mathrm{Ti}$ & - & 475 & $468[17]$ \\
\hline$v \mathrm{Ti}-\mathrm{O}-\mathrm{Ti}$ & - & 465 & $468[17]$ \\
\hline$\alpha-\mathrm{Fe}_{2} \mathrm{O}_{3}$ & & & $414[14]$ \\
\hline
\end{tabular}

Смещение характеристических колебаний связи Ті-О в сторону меньших частот при модифицировании рутила железом вызвано искажениями симметрии координационного окружения $\mathrm{Ti}^{+4}$ и является признаком присутствия в кристаллической структуре катионов $\mathrm{Fe}^{+3}$.

Результаты исследований получены на оборудовании Центра коллективного пользования научным оборудованием ВГУ.

\section{СПИСОК ЛИТЕРАТУРЫ}

1. Carneiroa J. O., Teixeiraa V., Portinhaa A., Magalhãe A., Coutinhob P., Tavaresa C. J., Newton R. // Materials Science and Engineering: B, 2007. vol. 138, № 8, pp. 144150.

2. FujishimaA., Honda K. // Nature, 1972, vol. 238, pp. $37-40$.

3. Zakrzewska K., Radecka M., Rekas M. // Thin Solid Films, 1997, vol. 310, pp. 161-166.

4. Al-Salim N. I., Bagshaw S. A., Bittar A., Kemmitt T., McQuillan A. J., Mills A. M., Ryan M. J. // J. Mater. Chem, 2000. vol. 10, pp. 2358-2363.

5. Sood S., Umar A., Mehta S. K., KansalS. K. // J. of Colloid and Interface Science. 2015, vol. 450, pp. 213223.

6. Kaleji B. K., Sarraf-Mamoory R. NakataK., Fujishima A. // J. of Sol-Gel Science and Technology November, 2011, vol. 60, pp. 99.

7. Li F. B., Li X. Z., Hou M. F.// Appl. Catal. B, 2004, vol. 48, № 3, pp. 185-194.
8. Wu Y., Lu G., Li S. // Catalysis Letters, 2009, vol. 133, pp. 97-105.

9. Неорганическая химия. Химия элементов, m. 1./ Ю. Д. Третьяков и др. М.: изд-во Моск. ун-та: Академкнига, 2007, с. 230, 421.

10. Ganesh I., KumarP. P., GuptaA. K., Sekhar P. S. C., Radha K., Padmanabham G., Sundararajan G. // Processing and Application of Ceramics, 2012, vol. 6, № 1, pp. 2136.

11. Hanaor D. A. H., AssadiM. H. N., Li S., Yu A., Sorrell C. C. // Computational Mechanics, 2012, vol. 50, pp. 185-194.

12. Wantala K., Tipayarom D., Laokiat L., Grisdanurak N. // Reaction Kinetics and Catalysis Letters, 2009, vol. 97. pp. 249-254.

13. Логачева В. А., Афонин Н. Н., Вахтель В. М., Киселева Ю. А., Сёмов Ю. Г. // Конденсированнье сре$\partial b l, 2016$, т. 18, № 3, с. 345-355.

14. Tolstoy V. P., et al. Handbook of Infrared Spectroscopy of Ultrathin Films. N. Y. Willey: 2003, pp. 671-673.

15. Давыдов А. А. ИК-спектроскопия в химии поверхности окислов. Изд-во: Наука, Новосибирск, 1984, c. 13-24.

16. Накамото К. ИК-спектры и КР-спектры неорганических и координационных соединений. М.: Мир, 1991, $536 \mathrm{c}$.

17. Vasconcelos D. C. L., Costa V. C., Nunes E. H. M., Sabioni A. C. S., Gasparon M., Vasconcelos W. L. // Materials Sciences and Applications, 2011, vol. 2, № 10, pp. 1375-1382.

18. Phillippi C. M., Lyon S. R. // Physical Review B, 1971, vol. 3, № 6, pp. 2086-2087. 


\title{
IR SPECTROSCOPY OF Fe-TiO, ${ }_{2}$ FILMS PREPARED BY MAGNETRON SPUTTERING
}

\author{
(ㄷ) 2017 V. A. Logacheva ${ }^{1}$, N. N. Afonin ${ }^{2}$, A. N. Lukin ${ }^{1}$, L. N. Nikitin ${ }^{3}$, Yu. A. Kiseleva ${ }^{1}$ \\ ${ }^{1}$ Voronezh State University, 1 Universitetskaya sq., 394018 Voronezh, Russia \\ e-mail: kcmf@vsu.ru \\ ${ }^{2}$ Voronezh Pedagogical University, 86 Lenin str., Voronezh 394043, Russia \\ e-mail:nafonin@vspu.ac.ru \\ ${ }^{3}$ Voronezh State Technical University, 14 Moscovsky av., Voronezh 394026, Russia
}

Received 17.04.2017

\begin{abstract}
The doping of $\mathrm{TiO}_{2}$ films with various metals, including $\mathrm{Fe}$, allows measuring the $\mathrm{TiO}_{2}$ band gap, which makes it possible to use the compounds for photovoltaic applications. The discussed research was aimed at studying the formation of complex oxides by IR spectroscopy on the surface and inside $\mathrm{Fe}_{-} \mathrm{TiO}_{2}$ films. The films were prepared by magnetron sputtering of iron on titanium oxide, followed by annealing in a diffractometer vacuum chamber at $P=1.33 \cdot 10^{-2} \mathrm{~Pa}$, vacuum reduction annealing at $\mathrm{P}=10^{-4} \mathrm{~Pa}$, and thermooxidation in flowing oxygen at atmospheric pressure.

The films were deposited on single-crystal silicon substrates with a Pt sublayer. Fe magnetron sputtering was conducted in a modernized vacuum unit UVN-1: the discharge was excited in $13.3 \cdot 10^{-}$ ${ }^{2} \mathrm{~Pa}$ high-purity argon. The discharge voltage was $420 \mathrm{~V}$. A metal iron target with an impurity level lower than 0.01 at.\% was used as a cathode. The sputtering rate was $7.2 \mathrm{~nm} / \mathrm{s}$. The thickness of the iron film was determined by the time of spluttering and was $120 \mathrm{~nm}$.

The phase composition of $\mathrm{Fe}^{-\mathrm{TiO}_{2}}$ films was studied by X-ray powder diffraction using ARL X'TRA diffractometer. The reflectance-absorption (RAS) and total internal reflection (ATR) spectra of the films were measured with the help of the Vertex-70 infrared Fourier spectrometer by Brooker (Germany). The reflectance-absorption spectra were measured with a mirror reflection attachment with a $13-83^{\circ}$ variable incidence angle. ATR spectra were measured with a single-pass Platinum-ATR with a diamond prism.

Films obtained under different annealing regimes were heterophase and contained: $\mathrm{TiO}_{2}$ in the rutile structure, two iron oxides $-\mathrm{Fe}_{3} \mathrm{O}_{4}$ and $\mathrm{Fe}_{2} \mathrm{O}_{3}$, and phases of complex oxides based on titanium and iron oxides: $\mathrm{FeTiO}_{3}$ (ilmenite) and $\mathrm{Fe}_{2} \mathrm{TiO}_{4}$ (ulvospinels).

The research demonstrated that the shift in the characteristic vibrations of the Ti-O bond toward lower frequencies can be explained by the presence of $\mathrm{Fe}^{+3}$ cations in the $\mathrm{TiO}_{2}$ crystal structure. It was established that the absorption bands caused by the deformation vibrations of the $\mathrm{Fe}-\mathrm{OH}$ bond can only be observed in ATR spectra and are shifted toward higher frequencies $\left(1.045 \mathrm{~cm}^{-1}\right)$ compared to individual iron oxides, which indicates the formation of complex oxides in the $\mathrm{Fe}^{-\mathrm{TiO}_{2}}$ films.

The shift of the characteristic vibrations of Ti-O bond toward the lower frequencies during the modification of rutile by iron is caused by distortions in the symmetry of the $\mathrm{Ti}^{+4}$ coordination environment and indicates the presence of $\mathrm{Fe}^{+3}$ cations in the crystal structure.
\end{abstract}

Keywords: IR spectroscopy, magnetron sputtering, $\mathrm{Fe}-\mathrm{TiO}_{2}$ film.

\section{ACKNOWLEDGMENTS}

The results of the research were obtained using the equipment of VSU's Equipment Centre for Collective Use of Scientific Equipment

\section{REFERENCES}

1. Carneiroa J. O., Teixeiraa V., Portinhaa A., Magalhãe A., Coutinhob P., Tavaresa C. J., Newton R. Materials Science and Engineering: B, 2007. vol. 138, no. 8, pp. 144-150. DOI: https://doi.org/10.1016/j.mseb.2005. 08.130
2. Fujishima A., Honda K. Nature, 1972, vol. 238, pp. 37-40. DOI:10.1038/238037a0

3. Zakrzewska K., Radecka M., Rekas M. Thin Solid Films, 1997, vol. 310, pp. 161-166. DOI: https://doi.org/10. 1016/S0040-6090(97)00401-X

4. Al-Salim N. I., Bagshaw S. A., Bittar A., Kemmitt T., McQuillan A. J., Mills A. M., Ryan M. J. J. Mater. Chem, 2000. vol. 10, pp. 2358-2363. DOI: 10.1039/ B004384M

5. Sood S., Umar A., Mehta S. K., KansalS. K. J. of Colloid and Interface Science. 2015, vol. 450, pp. 213-223. DOI: https://doi.org/10.1016/j.jcis.2015.03.018 
6. Kaleji B. K., Sarraf-Mamoory R. Nakata K., Fujishima A. J. of Sol-Gel Science and Technology November, 2011, vol. 60, pp. 99. DOI: 10.1007/s10971-011-2560-2

7. Li F. B., Li X. Z., Hou M. F. Appl. Catal. B, 2004, vol. 48, no. 3, pp. 185-194. DOI: https://doi.org/10.1016/j. apcatb.2003.10.003

8. Wu Y., Lu G., Li S. Catalysis Letters, 2009, vol. 133, pp. 97-105. DOI:10.1007/s10562-009-0165-y

9. Inorganic Chemistry. Chemistry of Elements, vol. 1. I Ed. by Yu. D. Tretyakovet al. Moscow, Akademkniga Publ., 2007, pp. 230, 421. (in Russia)

10. Ganesh I., Kumar P. P., Gupta A. K., Sekhar P. S. C., Radha K., Padmanabham G., Sundararajan G. Processing and Application of Ceramics, 2012, vol. 6, no. 1, pp. 21-36. DOI:10.2298/PAC1201021G

11. Hanaor D. A. H., Assadi M. H. N., Li S., Yu A., Sorrell C. C. Computational Mechanics, 2012, vol. 50, pp. 185-194. DOI:10.1007/s00466-012-0728-4

12. Wantala K., Tipayarom D., Laokiat L., Grisdanurak N. Reaction Kinetics and Catalysis Letters, 2009, vol. 97. pp. 249-254. DOI 10.1007/s11144-009-0045-x
13. Logacheva V. A., Afonin N. N., Wachtel V. M., Kiseleva Yu. A., Sëmov Yu. G. Condensed Matter and Interphase, 2016, vol. 18, no. 3, pp. 345-355. Available at: http://www.kcmf.vsu.ru/resources/t_18_3_2016_005. pdf

14. Tolstoy V. P., et al. Handbook of Infrared Spectroscopy of Ultrathin Films. N.Y. Willey: 2003, pp. 671673.

15. Davydov A. A. IR Spectroscopy in the Chemistry of the Oxide Surface. Novosibirsk, Science Publ., 1984, pp. 13-24. (in Russia)

16. Nakamoto K. Infrared and Raman Spectra of Inorganic and Coordination Compounds. Wiley, New York, 1986, $427 \mathrm{p}$.

17. Vasconcelos D. C. L., Costa V. C., Nunes E. H. M., Sabioni A. C. S., Gasparon M., Vasconcelos W. L. Materials Sciences and Applications, 2011, vol. 2, no. 10, pp. 13751382. DOI: $10.4236 / \mathrm{msa} .2011 .210186$

18. Phillippi C. M., Lyon S. R. Physical Review B, 1971, vol. 3, no. 6, pp. 2086-2087. DOI:https://doi.org/10.1103/ PhysRevB.3.2086
Логачева Вера Алексеевна - к. х. н., с. н. с., Воронежский государственный университет; тел.: +7(4732) 208445, e-mail: kcmf@vsu.ru

АфонинНиколайНиколаевич - д. х. н., профессор, Воронежский государственный педагогический университет; тел.: +7(473) 2208445, e-mail: nafonin@vspu. ac.ru

Лукин Анатолий Николаевич - к. ф. - М. н., доцент кафедры физики твердого тела и наноструктур, Воронежский государственный университет; тел.: +7(950) 7548707, e-mail: ckp_49@mail.ru

Киселева Юлия Александровна - магистрант химического факультета, Воронежский государственный университет; тел.: +7(919) 2317692, e-mail: yulechkakiseleva-93@mail.ru

Никитин Леонид Николаевич - к. т. н., доцент кафедры конструирования и производства радиоаппаратуры, Воронежский государственный технический университет, тел.: +7(473) 2437706, e-mail: 1.n.nikitin@ mail.ru
Logachova Vera A. - Cand. Sci. (Chem.), Researcher, Voronezh State University; ph.: +7(4732) 208445, e-mail: kcmf@vsu.ru

Afonin Nikolay N. - Dr. Sci. (Chem.), Professor, Voronezh State Pedagogical University; ph.: +7(473) 2208445 , e-mail: nafonin@vspu.ac.ru

Lukin Anatoly N. - Cand. Sci. (Phys.-Math.), Associate Professor of the Department of Solid State Physics and Nanostructures, Voronezh State University; ph.: +7(950) 7548707, e-mail: ckp_49@mail.ru

Kiseleva Julia A. - the competitor for science degree of Master Science in Chemistry, Faculty of Chemistry, Voronezh State University; ph.: +7 (919) 2317692, e-mail: yulechka-kiseleva-93@mail.ru

Nikitin Leonid N. - Cand. Sci. (Eng.), Assistant Professor of the Department of Design and Production of Radio Equipment, Voronezh State Technical University; ph.: +7(473) 2437706, e-mail: 1.n.nikitin@mail.ru 